\title{
Wet-Chemical Assembly of 2D Nanomaterials into Lightweight, Microtube-Shaped, and Macroscopic 3D Networks
}

\author{
Florian Rasch, ${ }^{\dagger}$ Fabian Schütt, ${ }^{*}{ }^{\dagger}$ (이 Lena M. Saure, ${ }^{\dagger, \perp}$ Sören Kaps, ${ }^{\dagger}$ Julian Strobel, ${ }^{\dagger}$
} Oleksandr Polonskyi, ${ }^{\S \odot}$ Ali Shaygan Nia," Martin R. Lohe, ${ }^{\| \odot ~ Y o g e n d r a ~ K . ~ M i s h r a, ~}{ }^{\#}$ Franz Faupel, ${ }^{\S}$ Lorenz Kienle, ${ }^{\ddagger}$ Xinliang Feng, ${ }^{\mathbb{O}}$ and Rainer Adelung ${ }^{\dagger}$

\author{
${ }^{\dagger}$ Chair for Functional Nanomaterials, Institute for Materials Science, ${ }^{\ddagger}$ Chair for Synthesis and Real Structure, Institute for Materials \\ Science, and ${ }^{\S}$ Chair for Multicomponent Materials, Institute for Materials Science, Kiel University, Kaiserstr. 2, 24143 Kiel, \\ Germany \\ ${ }^{\perp}$ Chair of Engineering Mechanics, Brandenburg University of Technology Cottbus-Senftenberg, Großenhainer Straße 57, 01968 \\ Senftenberg, Germany \\ "Department of Chemistry and Food Chemistry, Center for Advancing Electronics Dresden (cfaed), Technische Universität \\ Dresden, 01062 Dresden, Germany \\ \#NanoSYD, Mads Clausen Institute, University of Southern Denmark, Alsion 2, DK-6400 Sønderborg, Denmark
}

Supporting Information

\begin{abstract}
Despite tremendous efforts toward fabrication of three-dimensional macrostructures of two-dimensional (2D) materials, the existing approaches still lack sufficient control over microscopic (morphology, porosity, pore size) and macroscopic (shape, size) properties of the resulting structures. In this work, a facile fabrication method for the wet-chemical assembly of carbon $2 \mathrm{D}$ nanomaterials into macroscopic networks of interconnected, hollow microtubes is introduced. As demonstrated for electrochemically exfoliated graphene, graphene oxide, and reduced graphene oxide, the approach allows for the preparation of highly porous $(>99.9 \%)$ and lightweight $\left(<2 \mathrm{mg} \mathrm{cm}^{-3}\right)$ aeromaterials with tailored porosity and pore size as well as tailorable shape and size. The unique tubelike morphology with high aspect ratio enables ultralow-percolation-threshold graphene composites $\left(0.03 \mathrm{~S} \mathrm{~m}^{-1}, 0.05\right.$ vol\%) which even outperform most of the carbon nanotube-based composites, as well as highly conductive aeronetworks $\left(8 \mathrm{~S} \mathrm{~m}^{-1}, 4 \mathrm{mg} \mathrm{cm}^{-3}\right)$. On top of that, long-term

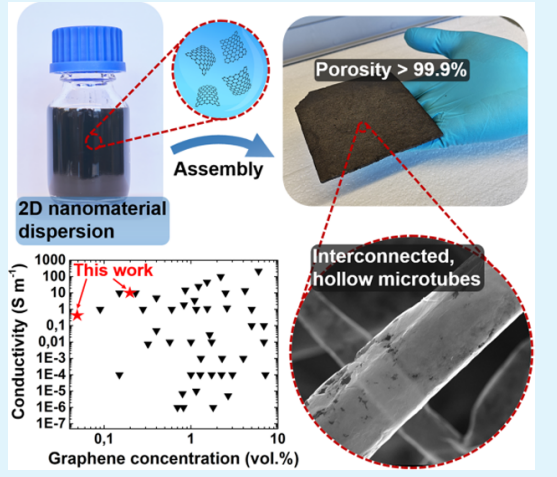
compression cycling of the aeronetworks demonstrates remarkable mechanical stability over 10000 cycles, even though no chemical cross-linking is employed. The developed strategy could pave the way for fabrication of various macrostructures of $2 \mathrm{D}$ nanomaterials with defined shape, size, as well as micro- and nanostructure, crucial for numerous applications such as batteries, supercapacitors, and filters.
\end{abstract}

KEYWORDS: 2D materials, assembly, hierarchical networks, graphene, polymer composites

\section{INTRODUCTION}

Since its discovery in 2004, ${ }^{1}$ the two-dimensional (2D) carbon nanomaterial (NM) graphene has attracted great attention owing to its extraordinary properties such as extremely high electron mobility, ${ }^{1-3}$ outstanding elasticity and stiffness, ${ }^{4}$ and exceptionally high thermal conductivity. ${ }^{5}$ To utilize these properties in practical applications (e.g., gas sensing, ${ }^{6,7}$ batteries, $^{8,9}$ supercapacitors ${ }^{1,11}$ ) on a large scale, however, it is crucial to develop methods for controlled, cost-effective assembly of single- or few-layer graphene sheets into macroscopic networks with defined morphology. Tremendous effort has already been put into fabrication of macrostructures of $2 \mathrm{D}$ carbon nanomaterials, ${ }^{12}$ and two main approaches, either based on dry or wet chemistry, have evolved in recent years. Dry-chemistry-based methods typically involve the use of nickel $(\mathrm{Ni})$ foams with fixed architecture (porosity, pore size) and geometry, which are converted into macroscopic, high-quality graphene foams using a chemical vapor deposition (CVD) process in the gas phase. ${ }^{13,14}$ Wet-chemistry-based methods comprise self-assembly of graphene sheets from the solution into macroscopic three-dimensional (3D) networks and offer scalability, high yield, and cost-effectiveness. ${ }^{12}$ Here, many promising routes have been explored, ${ }^{15,16}$ but the most common ones are hydrothermal/solvothermal-, ${ }^{17,18}$ crosslinking-, ${ }^{19,20}$ and chemical reduction ${ }^{21,22}$-based assemblies. Despite significant efforts toward the development of lowcost and high-yield techniques for fabrication of macroscopic networks with tailorable micro- and macrostructures, it remains a great challenge. The existing approaches still lag behind these complex requirements and meet them only

Received: September 20, 2019

Accepted: November 5, 2019

Published: November 5, 2019 

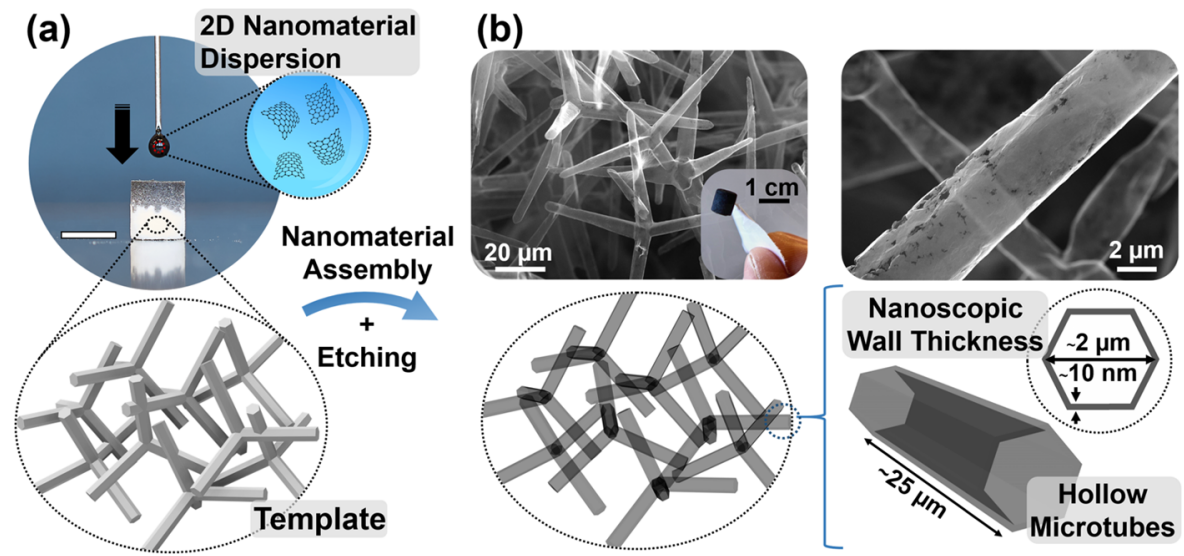

Figure 1. Wet-chemical assembly of graphene sheets into tubular, macroscopic 3D networks: (a) Infiltration of a highly porous and superhydrophilic template with an aqueous dispersion of graphene sheets, leading to a nanomaterial assembly on the surface of the template (scale bar: $6 \mathrm{~mm}$ ). (b) After wet-chemical etching of the ceramic template, the resulting macroscopic sample constitutes a highly porous (>99.9\%) network of interconnected, hollow microtubes with nanoscopic wall thickness. The inset photograph shows an ultralightweight graphene aeronetwork $\left(2 \mathrm{mg} \mathrm{cm}^{-3}\right)$ attached to a piece of paper.

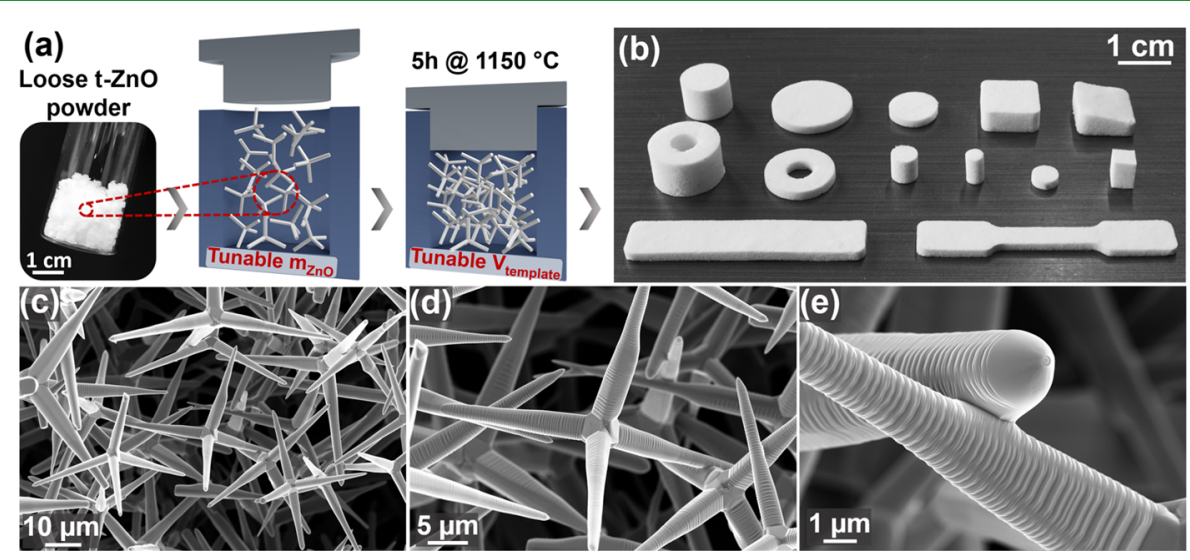

Figure 2. Large-scale fabrication of versatile templates: (a) Loose powder of tetrapodal $\mathrm{ZnO}(\mathrm{t}-\mathrm{ZnO})$ microparticles is pressed into tablets using a mold. Variation of $\mathrm{t}-\mathrm{ZnO}$ mass and template volume enables tuning of porosity up to $98 \%$. (b) High-temperature annealing results in macroscopic frameworks constituting interconnected microrods. Templates with various geometries can be fabricated by adjusting the shape of the mold. (c-e) Scanning electron microscopy (SEM) images of a ceramic template with $94 \%$ porosity, showing the freestanding network with interconnections among individual microrods formed after high-temperature annealing.

partially, e.g., defined morphology provided by $\mathrm{Ni}$ foams in dry-chemistry-based techniques, ${ }^{13}$ or low cost and high yield in wet-chemistry-based assemblies. ${ }^{21}$ Furthermore, the preparation of macrostructures with both tunable porosity and pore size, and tailored macroscopic geometries has not yet been realized and is of high importance for applications that require strict sample geometries and porosities, such as in the fields of energy storage, ${ }^{9,11}$ tissue engineering, ${ }^{23,24}$ and gas detection. ${ }^{6,25}$

The here introduced versatile strategy for the wet-chemical assembly of $2 \mathrm{D}$ carbon nanomaterials allows their shaping into cubic centimeter-sized, macroscopic networks of interconnected, hollow microtubes with a wall thickness well below 10 $\mathrm{nm}$. The fabrication process is based on a highly porous (up to 98\%) ceramic template composed of interconnected microrods (length $\sim 25 \mu \mathrm{m}$, diameter $\sim 1 \mu \mathrm{m}$ ), featuring a framework-like structure with open porosity and large free volume. Filling of the template with nanomaterial dispersions results in an assembly process during which the $2 \mathrm{D}$ nanomaterials uniformly coat the network of microrods. Removal of the template and critical point drying (CPD) yield freestanding and extremely lightweight $\left(\sim 2 \mathrm{mg} \mathrm{cm}^{-3}\right)$ carbon 3D networks.
To date, this counterintuitive effect of assembling nanomaterials wet-chemically into frameworks of interconnected microtubes is only known from one-dimensional structures like carbon nanotubes (CNTs). ${ }^{26}$ In this work, the process is demonstrated for different 2D nanomaterial systems, including electrochemically exfoliated graphene (EG), graphene oxide (GO), as well as reduced graphene oxide (rGO). Since the term "aerogel", which is commonly used for wet-chemically fabricated 3D carbon structures, is not appropriate for the developed networks, the terms "aeromaterials" and "aeronetworks" are interchangeably used in this work.

Even though no chemical cross-linking is employed during the fabrication, the tubular networks reveal high mechanical stability and conductivity while maintaining a high open porosity (>99.9\%). Long-term compression cycling of the three different carbon aeronetworks was performed, demonstrating high mechanical stability of Aero-GO and Aero-rGO over 10000 compression cycles at 33\% strain. Electrical characterization of prepared Aero-EG and EG composites with varying graphene concentration revealed high conductivity $(8$ $\mathrm{S} \mathrm{m}^{-1}$ at $4 \mathrm{mg} \mathrm{cm}^{-3}$ ) and an ultralow percolation threshold of 0.05 vol.\%. In contrast to other wet-chemical preparation 
methods, the here presented approach results in a defined and open-porous structure with an unique set of properties that usually can only be obtained by CVD processes. Additionally, the deployment of a template enables fabrication of framework-like structures that can be easily tailored on the macroscopic (dimensions, geometry) scale as well as on the microscopic (porosity, pore size) scale. This is crucial for the development of aeromaterials with tunable properties, which fulfill the complex requirements of the various potential applications, e.g., batteries, ${ }^{8,27}$ supercapacitors, ${ }^{9,11}$ gas detectors, $^{6,25}$ and $3 \mathrm{D}$ scaffolds for tissue regeneration. ${ }^{23,28}$

\section{RESULTS AND DISCUSSION}

Wet-Chemical Assembly of Graphene Sheets into Interconnected Microtubes. The general concept of this work for the fabrication of carbon $2 \mathrm{D}$ nanomaterial-based aeromaterials is illustrated in Figure 1. After wet-chemical infiltration of a superhydrophilic and open-porous template with an aqueous dispersion of graphene sheets (Figure 1a), the nanomaterials assemble uniformly on the surface of the entire template. As shown in Figure 1b, the subsequent wet-chemical template removal results in a macroscopic and highly porous (>99.9\%) network of interconnected, hollow graphene microtubes with nanoscopic wall thickness $(\sim 10 \mathrm{~nm})$. The extremely high porosity and associated low density $\left(<2 \mathrm{mg} \mathrm{cm}^{-3}\right)$ of the resulting structure are indicated in the inset of Figure $1 \mathrm{~b}$, which shows a graphene aeronetwork being only attached to the cellulose fibers of a piece of paper.

Versatile Ceramic Templates. The unique microtubebased morphology of the fabricated aeromaterials originates from the employed ceramic templates (Figure 2), which enable a precise control over porosity, pore size, and geometry of the final aeronetworks. The fabrication of these templates follows a bottom-up approach.

Using the so-called flame transport synthesis, ${ }^{29,30}$ a high yield of loose tetrapod-shaped zinc oxide $(\mathrm{t}-\mathrm{ZnO})$ powder (photograph in Figure 2a) is synthesized. Recently, this process has been upscaled, allowing for large-scale synthesis of $\mathrm{t}-\mathrm{ZnO}$, as shown in Figure S1 (Supporting Information). For template fabrication, a defined amount of $\mathrm{t}-\mathrm{ZnO}$ powder is then pressed into pellets with desired volume using a rigid mold, as schematically shown in Figure 2a. During the subsequent high-temperature annealing step ( $5 \mathrm{~h}$ at 1150 ${ }^{\circ} \mathrm{C}$ ), random connection points between the single tetrapod arms form and yield a freestanding, macroscopic network composed of interconnected $\mathrm{ZnO}$ tetrapods with enhanced mechanical stability. ${ }^{31}$ Combined with the large-scale synthesis of $\mathrm{t}-\mathrm{ZnO}$, a large variety of templates with different geometries and dimensions can be easily produced by changing the shape of the used mold. A selection of various template shapes is presented in Figure $2 \mathrm{~b}$. Besides the size and shape of the templates, the porosity (or density) can also be tailored from 50 up to $98 \%$ by changing the initial amount of $\mathrm{t}-\mathrm{ZnO}$ powder for a given volume of the template. This is demonstrated in Figure S2a-c (Supporting Information) by a representative selection of scanning electron microscopy (SEM) images of $t$ $\mathrm{ZnO}$ networks with increasing density, ranging from $0.3 \mathrm{~g}$ $\mathrm{cm}^{-3}$ (corresponding to $94 \%$ porosity) to $1.5 \mathrm{~g} \mathrm{~cm}^{-3}$ (73\% porosity). Accordingly, the pore size decreases with decreasing porosity of the template, while the pore size distribution remains narrow and cannot be tailored considerably. Exemplary, SEM images of a highly porous (94\%) $\mathrm{t}-\mathrm{ZnO}$ network are shown in Figure $2 \mathrm{c}-\mathrm{e}$ with increasing magnification. Since this structure represents a framework of randomly distributed, interconnected microrods with diameters of $1-3 \mu \mathrm{m}$ and lengths of $20-30 \mu \mathrm{m}$, it possesses a continuous and large free volume, which is accessible from the outside. In conjunction with the hydrophilic surface of $\mathrm{t}-\mathrm{ZnO}$, the network can be infiltrated and, thereby, completely filled with various $2 \mathrm{D}$ nanomaterial dispersions, which is essential for the here presented fabrication approach of carbon nanomaterial-based aeromaterials.

Template-Mediated Assembly of 2D Nanomaterials. The wet-chemical infiltration of the self-engineered $\mathrm{t}-\mathrm{ZnO}$ template and the associated assembly of $2 \mathrm{D}$ nanomaterials on the surface of the entire macroscopic framework are presented in Figure 3. In this simple deposition method, a dispersion of

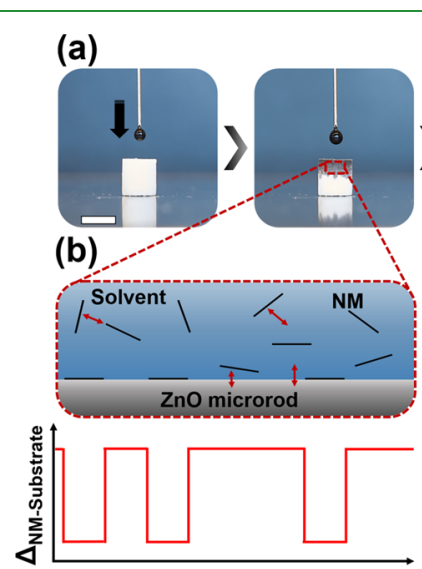

(d)

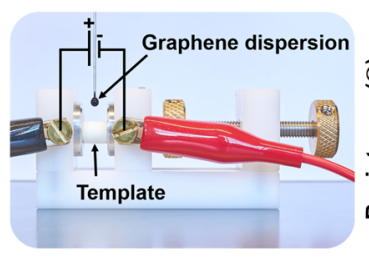

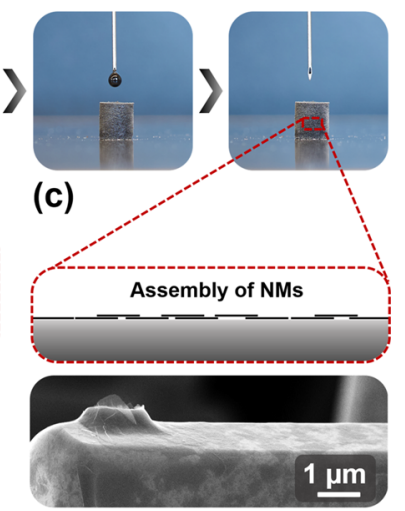

(e)

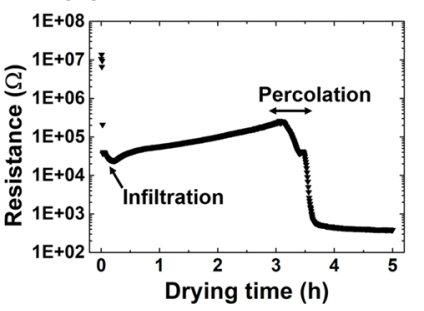

Figure 3. Template-mediated assembly of $2 \mathrm{D}$ carbon nanomaterials: (a) Sequence of photographs showing the infiltration of a $\mathrm{t}-\mathrm{ZnO}$ template with an aqueous dispersion of exfoliated graphene (EG). Scale bar: $6 \mathrm{~mm}$. (b) Schematic 2D model representing the resulting interactions during infiltration. As suggested in the graph, the interaction energy between nanomaterial (NM) and substrate $(\mathrm{ZnO}$ or NM) is locally decreased after the $\mathrm{NM}$ has attached to $\mathrm{ZnO}$. As a result, additional NMs favor attachment to other noncoated areas of the $\mathrm{ZnO}$ surface. (c) After infiltration and evaporation of the solvent, the nanomaterials assemble on the template surface, as shown in the SEM image for EG sheets on a $\mathrm{ZnO}$ microrod. (d) Photograph of the setup for in situ measurement of the electrical resistance of the $\mathrm{ZnO}$ template during infiltration with EG dispersion. (e) Resistance of the template during infiltration and subsequent drying process. After $\sim 3 \mathrm{~h}$ of medium evaporation, the resistance drops strongly by a few orders of magnitude, indicating the formation of conductive paths by assembly of graphene sheets on the template surface.

2D nanomaterials is dribbled on a highly porous $\mathrm{t}-\mathrm{ZnO}$ template, as shown for an aqueous EG dispersion in the sequence of photographs in Figure 3a. Due to the hydrophilic surface of $\mathrm{t}-\mathrm{ZnO}$ and the high porosity of the template, the water-based dispersion is soaked up readily and fills continuously more free volume of the network with progressing infiltration (Video S1, Supporting Information). 
Naturally, the maximum volume of infiltrated dispersion is equal to the free volume of the template.

After the network is filled with the nanomaterial dispersion, the solvent evaporates, which results in a uniform assembly of the nanomaterials on the surface of the $\mathrm{t}-\mathrm{ZnO}$ network. While the presented method represents a simple, yet efficient and homogeneous wet-chemical coating method, the underlying physicochemical mechanisms are more complex and require further clarification. Most importantly, the open framework structure of the $\mathrm{t}-\mathrm{ZnO}$ template enables filling of the entire free space and prevents filtering of the nanomaterials, which is the first key aspect toward homogeneous deposition of the nanomaterials on the template surface. Furthermore, as suggested in the schematic $2 \mathrm{D}$ model of a $\mathrm{ZnO}$ tetrapod arm after infiltration (Figure $3 \mathrm{~b}$ ), four different interactions may be considered to explain the assembly process of the $2 \mathrm{D}$ nanomaterials: solvent-nanomaterial (NM), NM-NM, t$\mathrm{ZnO}-\mathrm{NM}$, and $\mathrm{t}-\mathrm{ZnO}-$ solvent. First, the combination of solvent-nanomaterial and nanomaterial-nanomaterial interactions must allow for a stable and homogeneous dispersion of the nanomaterials to prevent agglomeration and achieve a uniform nanomaterial concentration within the entire network after infiltration. At this point, the entire free volume of the $t$ $\mathrm{ZnO}$ network is filled with a dispersion of nanomaterials and the solvent starts to evaporate continuously, leaving increasing free volume behind.

To ensure uniform assembly of the graphene sheets on the surface of the $\mathrm{t}-\mathrm{ZnO}$ network, the $\mathrm{t}-\mathrm{ZnO}$-nanomaterial interaction has to be higher than the nanomaterial-nanomaterial interaction. This is illustrated in the proposed schematic 2D model (Figure 3b) and the corresponding graph, which shows the interaction energy $\Delta_{\mathrm{NM} \text {-substrate }}$ between the nanomaterial and the substrate along the $\mathrm{ZnO}$ microrod. Upon attachment of a nanomaterial to the $\mathrm{t}-\mathrm{ZnO}$ surface, $\Delta_{\mathrm{NM} \text {-substrate }}$ is locally decreased. Therefore, the driving force for dispersed nanomaterials to attach to noncoated regions of the $\mathrm{t}-\mathrm{ZnO}$ surface is larger than the one for attachment to already deposited nanomaterials. Consequently, the nanomaterials assemble uniformly on the template surface and form a rather homogeneous film, as schematically shown in Figure 3c. This is confirmed on the representative SEM image in Figure $3 \mathrm{c}$, showing a $\mathrm{ZnO}$ microrod covered with a nanoscopic thin layer formed by assembly of EG sheets. Remarkably, the micrograph reveals conformation of the sheets even to the curved surface of the hexagonal $\mathrm{ZnO}$ tetrapod arm (Figure S3f, Supporting Information). Noteworthy, this uniform assembly indicates a relatively high interface stability between $\mathrm{ZnO}$ and the $2 \mathrm{D}$ nanomaterials, which is based on van der Waals interactions and attractive charge interactions between functional groups attached to the $2 \mathrm{D}$ materials and oxygen vacancies of the surface of $\mathrm{ZnO}$ (detailed discussion in the Supporting Information). Besides the wet-chemical infiltration with an EG dispersion, the process has also been adopted to a dispersion of GO sheets. Similarly, the $\mathrm{t}-\mathrm{ZnO}$ network is coated homogeneously with a thin layer of GO sheets, as shown in the SEM images in Figure S3 (Supporting Information).

To investigate the assembly process of the nanomaterials in more detail, the resistance of a $\mathrm{t}-\mathrm{ZnO}$ network was measured during the infiltration with an EG dispersion $\left(2 \mathrm{mg} \mathrm{mL}^{-1}\right)$ and during the subsequent evaporation of the solvent (water). As shown in Figure 3d, the cylindrical sample (height $=6 \mathrm{~mm}$, diameter $=6 \mathrm{~mm}$ ) was clamped between two electrodes and a constant voltage of $1 \mathrm{~V}$ was applied. The resulting graph is presented in Figure $3 \mathrm{e}$ and shows the measured resistance as a function of time. After an initial drop to about $10^{4} \Omega$ during the infiltration, the resistance increased slowly with progressing time and reached a maximum of ca. $10^{5} \Omega$ after $3 \mathrm{~h}$. Since the infiltration of a $\mathrm{t}-\mathrm{ZnO}$ network with deionized water yielded a smaller drop in resistance $\left(10^{5} \Omega\right.$, Figure S4 in the Supporting Information), the initial conductivity might be partially attributed to the EG sheets and the few ions which were present in the aqueous EG dispersion. The subsequent increase in resistance (from the start of infiltration to around $3 \mathrm{~h}$ ) can be explained with progressing evaporation of water and decreasing number of ions. However, after $3 \mathrm{~h}$, the resistance decreased drastically by 2 orders of magnitude over the course of $30 \mathrm{~min}$ and saturated at a value of $400 \Omega$. This measurement might suggest two conclusions regarding the wet-chemical assembly of the graphene sheets on the ceramic template. First, the graphene sheets might not cover the entire surface of the network immediately after infiltration as the contribution to the conductivity is mainly attributed to the water (ions). In other words, most of the graphene sheets stay most likely in dispersion during the infiltration and contribute only slightly to the conductivity. Second, with advancing evaporation of water, more graphene sheets may start to attach to the surface of the $\mathrm{t}-\mathrm{ZnO}$ network due to decreasing particle-substrate distance and increasing substrate-nanomaterial interaction. After $3 \mathrm{~h}$, the first conductive paths along the entire macroscopic network are formed, resulting in the steep resistance drop, which lasts for about $30 \mathrm{~min}$ (percolation region). A further discussion of the nanomaterial assembly process with a corresponding illustration (Figure S5) is presented in the Supporting Information.

Importantly, the demonstrated coating process is not limited to only one infiltration as shown in the example. In fact, several infiltrations can be carried out (followed by drying) and enable, in combination with the control over the concentration of the nanomaterial dispersion, a tunable coverage ranging from less than a monolayer up to several $100 \mathrm{~nm}$. Besides the applicability to other dispersions of nanomaterials, the presented method also allows the combination of different nanomaterials by successive or simultaneous infiltration.

Microtube-Shaped Aeronetworks of Carbon 2D Nanomaterials. The introduced macroscopic $\mathrm{t}-\mathrm{ZnO}$ framework does not only represent an ideal template for wetchemical coating with various $2 \mathrm{D}$ nanomaterials, but it can also be easily hydrolyzed by diluted acids. ${ }^{26}$ In fact, this allows for a facile fabrication of lightweight $\left(<2 \mathrm{mg} \mathrm{cm}^{-3}\right)$, macroscopic networks of hollow, interconnected microtubes composed of 2D nanomaterial assemblies, as demonstrated in Figure 4. By coating of the $\mathrm{t}-\mathrm{ZnO}$ networks with dispersions of $\mathrm{EG}$ or GO followed by wet-chemical removal of the sacrificial templates and critical point drying (CPD) of the structures, aeronetworks of EG (introduced as Aero-EG), GO (Aero-GO), and rGO (Aero-rGO) were fabricated. For preparation of Aero-rGO, an additional chemical reduction step in ascorbic acid was carried out to reduce GO prior to the template removal.

As confirmed by the photograph in Figure 4a, the shape and size of the $\mathrm{t}-\mathrm{ZnO}$ template were maintained for the three different aeronetworks. Furthermore, representative SEM images of Aero-rGO in Figure $4 b, c$ demonstrate perfect adoption of the tubular template morphology, while the highmagnification SEM images (Figure 4d,e) reveal the nanoscopic wall thickness of the microtubes. The latter can be easily tuned 


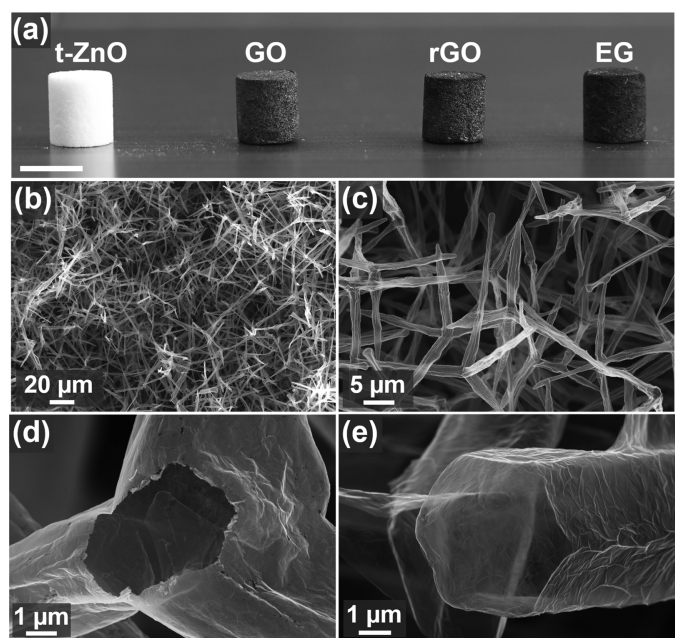

Figure 4. Aeronetworks of carbon $2 \mathrm{D}$ nanomaterials composed of hollow, interconnected microtubes: (a) Photograph of a sacrificial template $(\mathrm{t}-\mathrm{ZnO})$ and wet-chemically fabricated aeronetworks of graphene oxide (GO), reduced graphene oxide (rGO), and exfoliated graphene (EG). Scale bar: $1 \mathrm{~cm}$. (b-e) SEM images of an ultralightweight Aero-rGO network $\left(<2 \mathrm{mg} \mathrm{cm}^{-3}\right)$. (b, c) Lowermagnification images show perfect adoption of template morphology. (d, e) Higher-magnification images reveal nanoscopic wall thickness of the hollow microtubes.

between $\sim 25 \mathrm{~nm}$ up to a few $100 \mathrm{~nm}$ by increasing the number of wet-chemical infiltrations. Further reduction of the microtube wall thickness below $25 \mathrm{~nm}$ yielded aeromaterials with insufficient mechanical stability, indicating $\sim 25 \mathrm{~nm}$ as the critical thickness for mechanically stable networks. It should also be noted that even nanoscale surface features of $\mathrm{t}-\mathrm{ZnO}$ such as ripples (most likely due to step-edge diffusion during sintering of template) on the tetrapods (Figure 2e) are adopted and can be observed on the surface of the final hollow microtubes (Figure S6, Supporting Information), demonstrating a highly conformant assembly of the $2 \mathrm{D}$ nanomaterials on the surface of $\mathrm{t}-\mathrm{ZnO}$.
To date, wet-chemistry approaches for fabrication of $3 \mathrm{D}$ macroscopic structures of $2 \mathrm{D}$ nanomaterials mostly rely either on gelation of precursor solutions followed by freeze drying or $\mathrm{CPD}^{32,33}$ or on assembly of nanomaterials on sphere-based templates followed by template removal. ${ }^{34,35}$ As a result, the structures either lack sufficient control over morphology and porosity or possess partially closed pores with inaccessible free volume, respectively. Additionally, modification of the sample geometry and size remains a big challenge so far. Using the demonstrated wet-chemical infiltration method, these problems can be solved by employing large-scale fabricated sacrificial templates (Figure S7, Supporting Information) with tailorable shape, size, and porosity. For instance, the developed process enables the preparation of macroscopic aeromaterials with a highly complex shape such as hollow cylinders with a large aspect ratio (Figure S8, Supporting Information). Compared to other wet-chemically prepared 3D structures, the introduced aeronetworks also stand out due to their highly accessible framework-like structure with interconnected, hollow microtubes, making them attractive for a broad range of applications, ${ }^{36}$ e.g., gas sensing, ${ }^{6,25} 3 \mathrm{D}$ cell scaffolding, ${ }^{23,24}$ batteries (e.g., lithium-air ${ }^{27}$ ), supercapacitors, ${ }^{10,11}$ and energy harvesting and conversion. ${ }^{37}$

Besides the general feasibility of the presented method to fabricate macroscopic 3D networks of various $2 \mathrm{D}$ nanomaterials, it also allows the preparation of lightweight hybrid structures by combination of nanomaterials. For instance, wet-chemical infiltration of a $\mathrm{t}-\mathrm{ZnO}$ template with an aqueous dispersion of GO sheets and cellulose nanocrystals (CNC) followed by chemical reduction in ascorbic acid, template removal in diluted acid, and CPD yields a microtube-shaped network constituting a homogeneously interwoven layer of rGO and CNC (Figure S9, Supporting Information). Essentially, this can be further expanded to simultaneous or successive infiltration with different nanomaterial dispersions and thus enables a high degree of versatility and fabrication of 3D macroscopic networks with tailored properties for various applications. Furthermore, the aeronetworks can be employed (a)
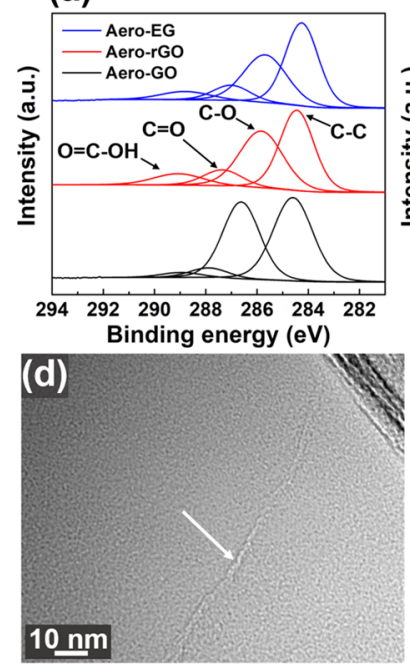

(b)
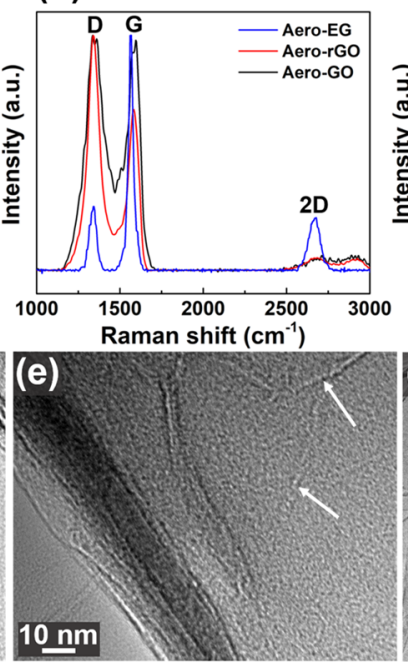

(c)
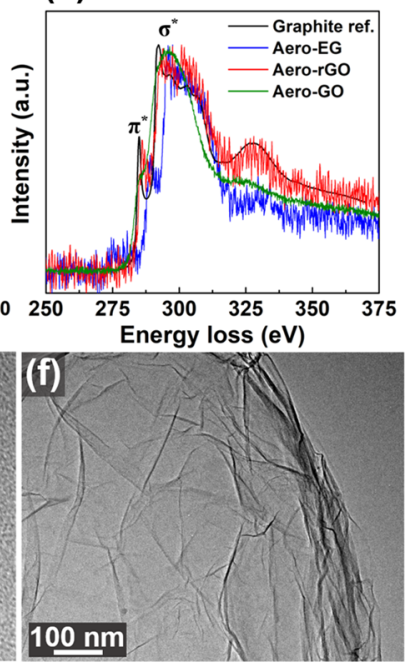

Figure 5. Structural and chemical characterization of aeronetworks: (a) XPS images of C 1s of Aero-GO, -rGO, and -EG networks, denoted with corresponding binding peaks. (b) Raman spectra of aeronetworks with respective vibration modes. (c) Low-loss electron energy-loss (EEL) spectra of Aero-EG, rGO, and graphite reference. (d-f) Transmission electron microscopy (TEM) images of Aero-GO revealing (d, e) seamlike joints (white arrows) indicating junctions between single rGO sheets and (f) the wrinkled surface morphology of the hollow tubes. 
(a)

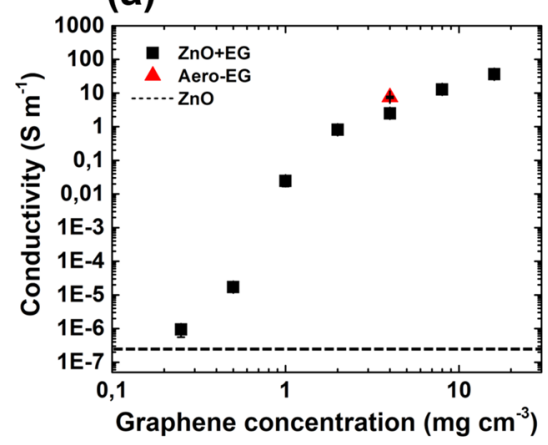

(b)

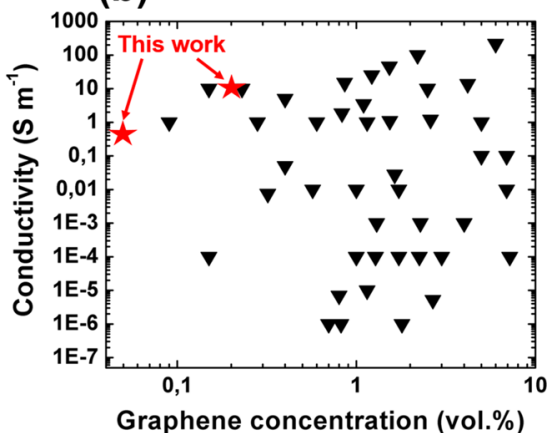

Figure 6. Electrical conductivity of macroscopic ZnO-EG composites and Aero-EG: (a) Conductivity of EG-coated t-ZnO networks as a function of EG concentration. Percolation within the $\mathrm{ZnO}$ network occurs at a low EG concentration of about $1 \mathrm{mg} \mathrm{cm}^{-3}$, which corresponds to a volume percentage of $0.05 \%$. The electrical conductivity of Aero-EG $\left(4 \mathrm{mg} \mathrm{cm}^{-3}\right)$ is presented as well. (b) Plot of the electrical properties of wet-chemically fabricated graphene-polymer composites, adapted from ref 54. The results for the EG composites from this work are indicated with the red stars. The list of all composites with corresponding references from this graph is shown in Table S1 (Supporting Information).

as templates for in situ growth of nanostructures on the surface of the microtube-based framework. As a proof of concept, Aero-GO was decorated with magnetic $\mathrm{CoFe}_{2} \mathrm{O}_{4}$ nanoparticles (NPs) via direct wet-chemical synthesis of the NPs on the template. The high coverage of the lightweight $\left(<2 \mathrm{mg} \mathrm{cm}^{-3}\right)$ framework (SEM images in Figure S10, Supporting Information) allows guided movement of the aeromagnet by a permanent magnet, as demonstrated in Video S2 in the Supporting Information.

Structural and Chemical Characterization. Further structural and chemical characterization of the fabricated aeronetworks is presented in Figure 5. X-ray photoelectron spectroscopy (XPS) measurements of Aero-GO (Figure 5a) reveal four peaks located at 284.6, 286.6, 287.8, and $289.0 \mathrm{eV}$, corresponding to $\mathrm{C}=\mathrm{C} / \mathrm{C}-\mathrm{C}$ in aromatic rings, $\mathrm{C}-\mathrm{O}$ (alkoxy and epoxy), $\mathrm{C}=\mathrm{O}$, and $\mathrm{COOH}$ groups. ${ }^{38}$ As expected, the pronounced $\mathrm{C}-\mathrm{O}$ peak reflects the large fraction of oxygencontaining functional groups in Aero-GO. After reduction in ascorbic acid for $48 \mathrm{~h}$, the intensity of the $\mathrm{C}-\mathrm{O}$ peak was clearly decreased for Aero-rGO (Figure 5a), revealing partial reduction of Aero-GO. Further evidence for successful chemical reduction via ascorbic acid is the color change of the sample from brown (Aero-GO) to dark (Aero-rGO), as also shown in Figure 4a. Similarly, the XPS images for AeroEG (Figure 5a) show a decreased intensity of the $\mathrm{C}-\mathrm{O}$ peak compared to Aero-GO, which indicates a smaller fraction of oxygen-containing functional groups. However, the sample seems to contain a considerable amount of oxygen, which might be related to the preparation of the graphene dispersion via electrochemical exfoliation. During this process, ${ }^{39}$ partial oxidation of graphite by $\mathrm{OH}$ radicals occurs, leading to a slight oxidation of the final graphene sheets especially on the edges of the flakes.

In addition to the XPS measurements, the aeronetworks were also characterized by Raman spectroscopy using a 532.2 $\mathrm{nm}$ laser with a spot size of $\sim 1.41 \mu \mathrm{m}$. As shown in Figure S11 (Supporting Information), the characteristic $\mathrm{t}-\mathrm{ZnO}$ modes do not appear in the spectra of the three aeronetworks, emphasizing complete removal of the sacrificial template. In the Raman spectra of Aero-GO, Aero-rGO, and Aero-EG (Figure $5 \mathrm{~b}$ ), three main bands can be observed: The $\mathrm{D}$ band $\left(1340 \mathrm{~cm}^{-1}\right)$ which corresponds to the breathing mode of $\mathrm{sp}^{2}$ carbon atoms in aromatic rings and is only activated in the presence of disorder; the $G$ band $\left(1580 \mathrm{~cm}^{-1}\right)$ which originates from in-plane bond-stretching motion of pairs of carbon $\mathrm{sp}^{2}$ carbon atoms; and the $2 \mathrm{D}$ band $\left(2680 \mathrm{~cm}^{-1}\right.$, also called $\mathrm{G}^{\prime}$ band) which is the second order of zone-boundary phonons. ${ }^{40,41}$ Since the $\mathrm{D}$ band is especially prominent in the Raman spectra of Aero-GO and Aero-rGO, a relatively high degree of disorder is present in these aeronetworks. This results from symmetry breaking in the carbon honeycomb lattice, most likely induced by functionalization of graphene with various oxygen-containing groups and the associated change in carbon hybridization from $\mathrm{sp}^{2}$ to $\mathrm{sp}^{3.42,43}$ In comparison to Aero-GO and Aero-rGO, the $\mathrm{D}$ band of Aero-EG is clearly less pronounced, accompanied by a lower intensity ratio of $\mathrm{D}$ and $\mathrm{G}$ bands. This indicates a considerably lower degree of defects in Aero-EG. It should be noted that the $\mathrm{D}$ band may also be partially attributed to edges of the graphene (oxide) sheets since the spot size of the laser $(\sim 1.41$ $\mu \mathrm{m})$ is larger than the average lateral size of the GO and EG sheets $(\sim 500 \mathrm{~nm})$. In addition to the strong $\mathrm{D}$ band, the Raman spectra (Figure $5 \mathrm{~b}$ ) reveals a decrease in the intensity ratio of the $\mathrm{D}$ and $\mathrm{G}$ band for Aero-rGO compared to AeroGO. This may not be expected as the partial elimination of functional groups should lead to partial restoration of $\mathrm{sp}^{2}$ hybridization and, thus, reduction of disorder. However, a similar trend after reduction of GO has also been reported in several other works, which is mostly explained by a numeric increase of $\mathrm{sp}^{2}$ domains at smaller overall size. ${ }^{44,45}$ On the other hand, it is argued that the intensity ratio of $D$ and $G$ bands cannot be compared between the two states as only rGO corresponds to a graphene-like state while GO is in an amorphous state. ${ }^{44,46,47}$ Finally, the Raman spectra also reveal that the intensity ratio of the $2 \mathrm{D}$ band and the band at 2940 $\mathrm{cm}^{-1}$ is considerably increased for Aero-EG compared to AeroGO and Aero-rGO, which indicates a higher degree of graphitization. ${ }^{44,48}$

To investigate the electronic structure of the fabricated aeronetworks, electron energy-loss spectroscopy (EELS) measurements were performed on the samples (Figure $5 \mathrm{c}$ ). Compared to the graphite reference, ${ }^{49}$ the spectra of Aero-EG and Aero-rGO also exhibit the characteristic $\sigma^{*}$ and $\pi^{*}$ peaks (denoted in Figure 5c)..$^{50}$ However, the latter is less pronounced and, thus, indicates a smaller fraction of $\mathrm{sp}^{2}$ hybridization compared to graphite (or graphene) due to partial breaking of the aromatic ring structure by chemically attached functional oxygen-containing groups. As expected, the 
(a)

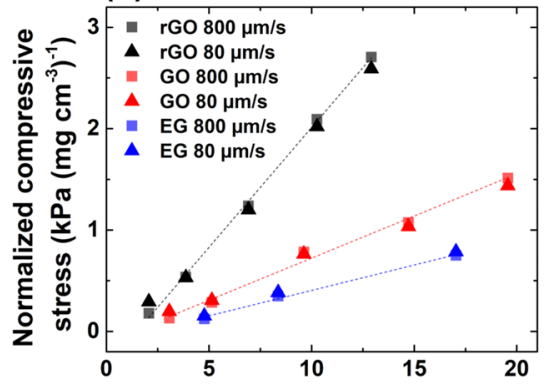

(c)

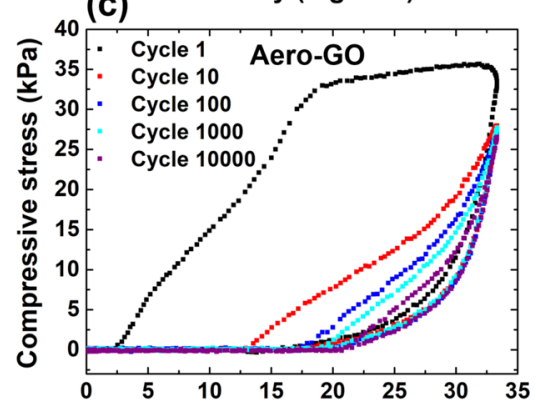

(e) Compressive strain (\%)

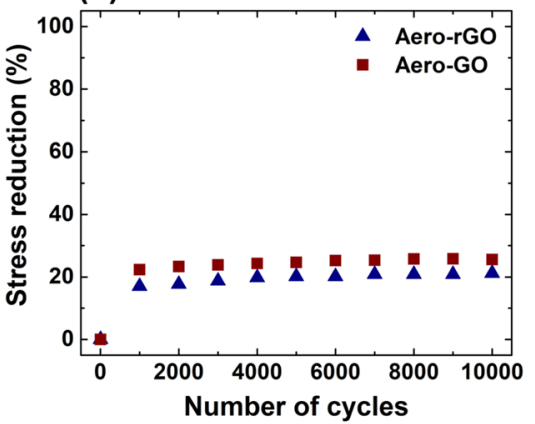

(b)

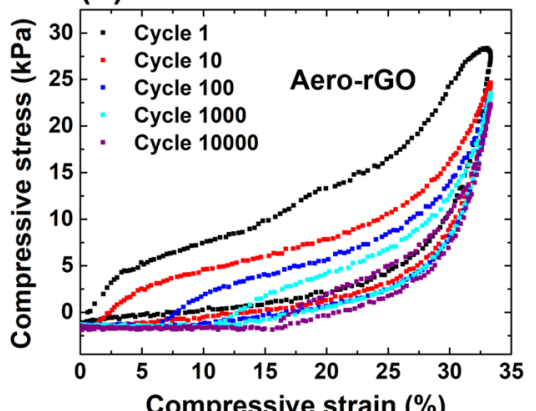

(d)

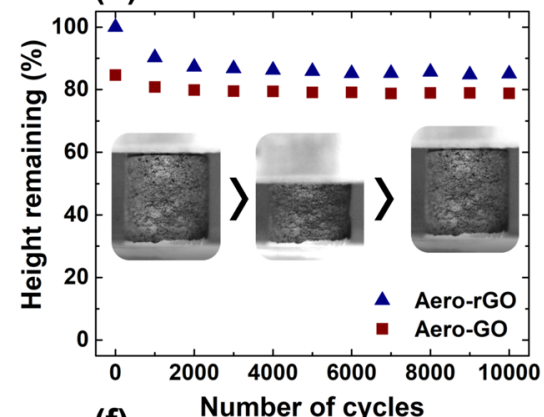

(f) Number of cycles

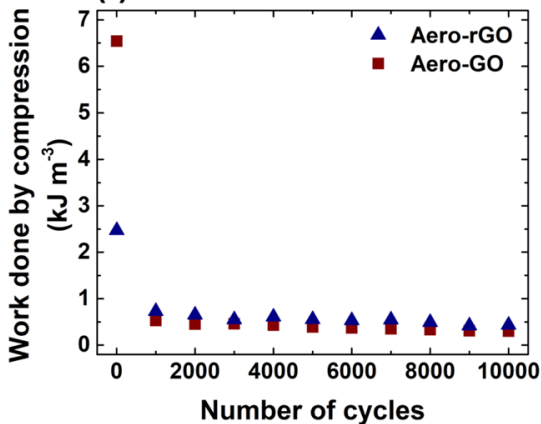

Figure 7. Long-term compression cycles of carbon aeronetworks: (a) Normalized compressive stress (at 7\% strain) versus density for Aero-GO, -rGO, and -EG at different deformation rates. Stress-strain curves of (b) Aero-rGO $\left(14 \mathrm{mg} \mathrm{cm}^{-3}\right)$ and (c) Aero-GO $(18 \mathrm{mg} \mathrm{cm}$ ) for up to 10000 compression cycles. (d) Height retention, (e) reduction of maximum compressive stress, and (f) work done by compression of Aero-GO and Aero-rGO during repeated compression at a strain of 33\%. The inset photographs in (e) show one compression cycle of Aero-rGO (14 mg $\mathrm{cm}^{-3}$ ) at $33 \%$ strain with complete recovery.

$\pi^{*}$ peak is even less distinct for Aero-GO since this sample possesses the highest concentration of functional groups, resulting in a lower percentage of $\pi$ bonds compared to AerorGO and Aero-EG. Furthermore, the wall thickness of the hollow interconnected microtubes was determined by means of the log-ratio method, ${ }^{51}$ yielding an average thickness of $\sim 25$ $\mathrm{nm}$ for all three aeronetworks (density $<2 \mathrm{mg} \mathrm{cm}^{-3}$ ). This result confirms the successful fabrication of hierarchical, highly porous ( $>99.9 \%)$ networks which are composed of hollow microtubes with nanoscopic wall thickness. The transmission electron microscopy (TEM) image of Aero-GO (Figure 5d) reveals a wrinkled surface of the hollow GO tube, which has also been found for Aero-rGO and Aero-EG and is a characteristic feature for wet-chemically assembled networks. ${ }^{18,21,52}$ Additionally, the high-magnification micrographs in Figure 5e,f show seamlike defects (marked by arrows), which may represent junctions between single GO sheets. ${ }^{53}$

Conductivity of 3D Graphene Networks. Since the prepared EG networks exhibit the highest conductivity compared to the GO and rGO frameworks, their electrical properties were characterized in more detail. In particular, several $\mathrm{t}-\mathrm{ZnO}$ templates (porosity $\sim 94 \%$ ) were coated with increasing graphene concentration (ranging from 0.25 to 16 $\mathrm{mg} \mathrm{cm} \mathrm{cm}^{-3}$ ) by changing either the concentration of EG dispersion or the number of wet-chemical infiltrations. Afterwards, the resistance of these networks was measured (see Methods for more details), giving rise to the calculated conductivity as a function of the graphene concentration in Figure 6a.

While the networks are not conductive for graphene concentrations below $\sim 0.5 \mathrm{mg} \mathrm{cm} \mathrm{cm}^{-3}$, a strong increase in conductivity by several orders of magnitude occurs at $\sim 1 \mathrm{mg}$ $\mathrm{cm}^{-3}$, indicating percolation by formation of conductive pathways within the entire macroscopic network. In other words, the highly porous framework becomes already conductive at a threshold concentration as low as 0.05 vol\% of EG. To put this result in perspective, the plot in Figure $6 \mathrm{~b}$ compares values obtained for the composites presented here with the ones for other wet-chemically prepared graphenepolymer composites. To the best of our knowledge, the percolation threshold of $0.05 \mathrm{vol} \%$ is one of the lowest ever reported for graphene-based composites fabricated by means 
of wet chemistry. On top of that, the here presented networks have a remarkably high conductivity at relatively low EG concentrations, e.g., $8 \mathrm{~S} \mathrm{~m}^{-1}$ for Aero-EG with a density of 4 $\mathrm{mg} \mathrm{cm}{ }^{-3}$. Astonishingly, the EG composites even outperform most of the wet-chemically prepared CNT-polymer composites $^{55}$ despite the commonly larger aspect ratios of CNTs. The ultralow percolation threshold and high conductivity can be attributed to the unique morphology of the $\mathrm{t}-\mathrm{ZnO}$ networks, consisting of interconnected microrods which represent highly efficient conductive pathways with extremely high aspect ratios after deposition of conductive $2 \mathrm{D}$ nanomaterials. Therefore, a low coverage of conductive nanomaterials (i.e., EG sheets) is sufficient to reach the percolation threshold, while most other wet-chemical fabrication methods require much higher graphene loadings as they commonly rely on direct dispersion of $\mathrm{GO}$ sheets in the polymer matrices followed by reduction, resulting in larger fractions of isolated graphene sheets which cannot contribute to electrical conduction. ${ }^{56,57}$ Considering these remarkable electrical properties in combination with the large accessible surface area, the aeronetworks are also promising candidates for applications in the field of electrochemical energy storage, enabling fast charge-discharge rates. ${ }^{58}$ It should be noted that the aeromaterials could reach extremely high gravimetric capacities, but generally pose a challenge of reduced volumetric capacity due to the low tap density. To tackle this problem, the density of the aeromaterials could be increased by compression or by decreasing the porosity of the employed $\mathrm{t}-\mathrm{ZnO}$ templates. Finally, the $\mathrm{t}-\mathrm{ZnO} / \mathrm{EG}$ networks can easily be processed into graphene-polymer composites, e.g., by infiltration with poly(dimethylsiloxane) followed by curing and removal of $t$ $\mathrm{ZnO}$ in diluted acid (to be discussed in more detail in a separate work).

Long-Term Compression Cycling of Aeronetworks. To investigate the mechanical stability of the aeronetworks, compression cycling tests were carried out with up to 10000 cycles. Figure 7a shows the normalized compressive stress (at $7 \%$ strain) for Aero-rGO, Aero-GO, and Aero-EG as a function of the density of the networks. Evidently, the compressive stress increases linearly with the density of the samples, indicating increasing stiffness of the macroscopic networks originating from rising wall thickness of the hollow microtubes. Furthermore, altering the deformation rate by 1 order of magnitude $\left(80-800 \mu \mathrm{m} \mathrm{s}^{-1}\right)$ did not cause any significant change in compressive stress, which confirms similar observations reported previously. ${ }^{15,59}$ The plot in Figure $7 \mathrm{a}$ also reveals a clear difference in the slope of the linear fits for the three different aeronetworks, with the steepest one corresponding to Aero-rGO, followed by Aero-GO and Aero-EG. The lowest mechanical strength of Aero-EG might be related to a slightly less uniform assembly of the graphene sheets on the sacrificial template compared to the GO sheets, resulting in a lower mechanical stability of the assembled layer. The distinctly higher compressive strength of Aero-rGO compared to Aero-GO is most likely linked to increased van der Waals interaction between the rGO sheets after the chemical reduction of GO in ascorbic acid since the preceding wet-chemical infiltration and the resulting assembly process of the GO sheets are same for Aero-GO and Aero-rGO.

The observed differences in compressive strength between the three aeronetworks are also reflected in the results of compression cycling tests, which were carried out on the samples for up to 10000 cycles and 33\% compressive strain.
While Aero-EG already collapses after the first cycles, AerorGO (14 $\mathrm{mg} \mathrm{cm}^{-3}$, Figure $\left.7 \mathrm{~b}\right)$ and Aero-GO $\left(18 \mathrm{mg} \mathrm{cm}^{-3}\right.$, Figure $7 \mathrm{c}$ ) demonstrate mechanical stability during the longterm cycling (compression of Aero-rGO in Video S3, Supporting Information), albeit the aeronetworks do not completely recover after 10000 cycles (height retention in Figure $7 \mathrm{~d}$ ). The stress-strain curves show hysteresis loops which indicate the viscoelastic properties of the aeronetworks, a typical phenomenon observed for almost all compressible carbon foams. ${ }^{60,61}$ In principle, the mechanical response during the loading process is characterized by two stages: a linearelastic regime corresponding to elastic buckling at the central joint of the hollow tetrapods or along the tetrapod arms and a nonlinear regime with increasing slope due to densification of the network. In fact, in situ SEM measurements demonstrate either bending of a hollow rGO microtube at the central joint (Figure S12 and Video S4, Supporting Information) or bending of the hollow tube itself (Figure S13 and Video S5, Supporting Information) induced by a micromanipulator, followed by fast recovery to the original state. Similar mechanisms have already been reported by Falvo et al. ${ }^{62}$ for multiwalled CNTs and have previously been shown for similar tubular structures grown by CVD. ${ }^{63}$ To achieve elastic recovery after compression, the deformation of the microtube-shaped network (i.e., buckling/bending of tubes) must be reversible. In other words, during unloading, the stored elastic energy has to overcome the intersheet van der Waals adhesion, ${ }^{15,64}$ acting, e.g., between bent hollow microtubes. It is suggested that the elastic restoring force of the tubular AerorGO and Aero-GO networks is not only enhanced with increasing wall thickness of the microtubes but also by the formation of nanosails between individual tubes (Figure S14, Supporting Information). As observed in the experiments, these nanosails occur increasingly for growing network density and arise from the assembly process of the GO sheets (for a more detailed discussion of the assembly process, see the Supporting Information). However, the graph in Figure $7 \mathrm{~d}$ reveals that the deformation of Aero-rGO and Aero-GO is not purely elastic but also contains a plastic contribution, indicated by the decreasing height retention with the number of compression cycles. After a considerable decrease during the first 2000 cycles, the aeronetworks stabilize and retain 55\% (Aero-rGO) and 37\% (Aero-GO) of their original height after 10000 cycles at $33 \%$ compressive strain. Plastic deformation may occur by buckling and bending of the hollow microtubes, which can ultimately lead to the formation of cracks at the joints (Figure S12e, Supporting Information) or pinning of the deformed state by van der Waals adhesion. The initial decrease in the maximum compressive stress by about $20 \%$ of the aeronetworks after 1000 cycles (Figure 7e) confirms the assumption that most of the plastic deformation occurs within the first 1000-2000 cycles. However, it should be emphasized that the plastic deformation is not necessarily irreversible since the duration of half a compression cycle might not be sufficient to allow complete recovery of all pinned states to their original states.

As shown in Figure $7 f$, the work done by compression drops strongly between the first and the 1000th cycle (from 2.5 to $0.75 \mathrm{~kJ} \mathrm{~m}^{-3}$ ). This might be related to the discussed partial plastic deformation of the macroscopic aeronetworks, leading to decreased height of the samples and less required work for further compression cycles. Recently, Wang et al. ${ }^{65}$ have studied the energy dissipative mechanisms for graphene foams 
experimentally and theoretically on the scale of graphene sheets and proposed that the mentioned buckling and bending of the hollow microtubes is essentially the result of rippling, sliding, and impacting of the constituent sheets. ${ }^{65}$

Despite the incomplete recovery, the aeronetworks demonstrate a remarkable mechanical stability sustaining 10000 compression cycles at relatively large strain, especially given that the lightweight frameworks (>99\% porosity) are prepared wet-chemically by assembly of individual $2 \mathrm{D}$ nanomaterials, which only interact via van der Waals forces. In contrast to many other existing approaches, ${ }^{61,64,66}$ no further chemical modification such as cross-linking was employed to enhance the mechanical properties.

\section{CONCLUSIONS}

A simple, versatile, as well as scalable method for the wetchemical assembly of 2D carbon nanomaterials into microtubebased, macroscopic 3D networks with tailorable density, porosity (up to 99.9\%), shape, and size was introduced. Owing to its simplicity, the approach also allows for a combination of various nanomaterials to form freestanding, lightweight $\left(<2 \mathrm{mg} \mathrm{cm}^{-3}\right)$ hybrid networks. Electrical characterization of the networks revealed an ultralow percolation threshold of the composites (0.05 vol\%) and notably high conductivity $\left(8 \mathrm{~S} \mathrm{~m}^{-1}\right.$ at $\left.4 \mathrm{mg} \mathrm{cm}^{-3}\right)$, which even outperform most CNT-polymer composites in terms of electrical properties. This represents a big advantage compared to CNT-based composites given the generally reported higher biocompatibility of graphene, making them also attractive for biomedical applications. Long-term compression cycling of the aeronetworks demonstrates their remarkable fatigue resistance sustaining 10000 compression cycles, even though no chemical cross-linking is employed. Considering the constantly improving synthesis methods of $2 \mathrm{D}$ nanomaterial dispersions, the presented approach could be adapted to other 2D nanomaterials such as transition-metal dichalcogenides or MXenes and pave the way for fabrication of a large variety of macroscopic aeronetworks with tailorable properties. This is highly relevant for various applications such as batteries, supercapacitors, 3D cell scaffolds, gas sensors, and filters, which require macroscopic sample dimensions with a defined shape, size, as well as micro- and nanostructures.

\section{METHODS}

Fabrication of Carbon 2D Nanomaterial Dispersions. Graphene oxide (GO) was prepared according to previously reported procedures. ${ }^{67,68}$ Afterward, the as-produced GO was dispersed in water via tip sonication to achieve homogeneous and stable dispersions. Aqueous dispersions of electrochemically exfoliated graphene (EG) were kindly provided by Sixonia Tech GmbH and used without further modification.

Fabrication of Aeromaterials. First, tetrapodal zinc oxide ( $t$ $\mathrm{ZnO})$ microparticles were prepared using the flame transport synthesis, which is described in detail elsewhere. ${ }^{29,31}$ In brief, a mixture of poly(vinyl butyral) and zinc powder with a grain size of 1$5 \mu \mathrm{m}$ (mass ratio, 2:1) was heated in a muffle furnace for $30 \mathrm{~min}$ at $900{ }^{\circ} \mathrm{C}$ (heating rate, $60{ }^{\circ} \mathrm{C} \mathrm{min}^{-1}$ ). The obtained loose $\mathrm{t}-\mathrm{ZnO}$ powder was pressed into cylindrical pellets $(6 \mathrm{~mm}$ diameter, $6 \mathrm{~mm}$ height) and sintered at $1150{ }^{\circ} \mathrm{C}$ for $5 \mathrm{~h}$, resulting in a network of interconnected $\mathrm{ZnO}$ tetrapods. To coat the $\mathrm{t}-\mathrm{ZnO}$ template with the carbon $2 \mathrm{D}$ nanomaterials, aqueous dispersions of exfoliated graphene (EG) or graphene oxide (GO) (each $2 \mathrm{mg} \mathrm{mL}^{-1}$ ) were dribbled on the ceramic template ( $94 \%$ porosity) to fill the free volume until no further dispersion was taken up. Afterward, the infiltrated $\mathrm{t}-\mathrm{ZnO}$ network was dried at $50{ }^{\circ} \mathrm{C}$ for $4 \mathrm{~h}$ to allow evaporation of the solvent (water) and simultaneous deposition of the nanomaterials on the surface of the template. For preparation of a $\mathrm{t}-\mathrm{ZnO}$ network coated with reduced GO (rGO), GO sheets are first deposited on the template and subsequently reduced by immersion in diluted Lascorbic acid $\left(0.1 \mathrm{mg} \mathrm{mL}^{-1}\right)$ for $24 \mathrm{~h}\left(\right.$ at $\left.50{ }^{\circ} \mathrm{C}\right)$. Then, the sample was washed thoroughly in deionized water. After deposition of the respective nanomaterials, the sacrificial template was etched wetchemically in diluted $\mathrm{HCl}(1 \mathrm{M})$ for $24 \mathrm{~h}$, washed in water and absolute ethanol, and dried by critical point drying using an EMS 3000 .

Characterization of t-ZnO Networks and Aeromaterials. SEM analysis was performed with a Zeiss Supra 55VP. For TEM characterization, an FEI Tecnai F30 G2 STwin was used. The macroscopic aeromaterials were carefully tapped with a TEM grid to transfer individual microtubes onto the grid. The electronic structure was characterized by electron energy-loss spectroscopy (EELS) using a GIF Quantum/Enfina energy analyzer. Raman spectra were recorded using an alpha300 RA (WITec) microscope with a triple grating spectrometer $\left(600 \mathrm{~g} \mathrm{~mm}^{-1}\right)$ and a charge-coupled device detector. The excitation wavelength of the laser was $532.2 \mathrm{~nm}$, and the laser spot size on the sample was $\sim 1.41 \mu \mathrm{m}$ with a maximum power of $52 \mathrm{~mW}$. XPS (Omicron Nanotechnology $\mathrm{GmbH}$ ) spectra were charge-referenced based on the $\mathrm{C} 1 \mathrm{~s}$ line of aliphatic carbon at $285.0 \mathrm{eV}$ and analyzed using the software CasaXPS.

For electrical characterization of the $\mathrm{t}-\mathrm{ZnO} / \mathrm{EG}$ networks and Aero-EG, the samples were mounted between two aluminum electrodes (Figure 3d) and conductive silver paste was used to enhance the electrical contact. The current-voltage characteristics were recorded using a Keithley 6400 sourcemeter in the two-wire sensing mode. For the in situ measurement of the resistance during infiltration of a $\mathrm{t}-\mathrm{ZnO}$ network with an $\mathrm{EG}$ dispersion, the $\mathrm{t}-\mathrm{ZnO}$ was first mounted between the electrodes as described above. Then, a constant voltage of $1 \mathrm{~V}$ was applied and the current was measured during the subsequent infiltration process. The resistance was calculated from the obtained data.

Compression cycling of the aeromaterials was performed using a self-built setup consisting of a Märzhäuser Wetzlar HS 6.3 micromanipulator and a force sensor (burster, type 9235/36), which were controlled by a self-written LabView program.

\section{ASSOCIATED CONTENT}

\section{Supporting Information}

The Supporting Information is available free of charge on the ACS Publications website at DOI: 10.1021/acsami.9b16565.

Upscaled synthesis of $\mathrm{t}-\mathrm{ZnO}$ powder (Figure $\mathrm{S} 1$ ); tunable template density (Figure S2); wet-chemical assembly of $2 \mathrm{D}$ carbon nanomaterials on ceramic template (Figure S3); resistance of $\mathrm{t}-\mathrm{ZnO}$ template during infiltration (Figure S4); assembly process of 2D carbon nanomaterial (Figure S5); adaption of template surface topography (Figure S6); photograph of a macroscopic and highly porous (>99\%) Aero-rGO sample (Figure S7); macroscopic aeromaterials with complex shape (Figure S8); combination of nanomaterials (Figure S9); functionalization of Aero-GO with $\mathrm{CoFe}_{2} \mathrm{O}_{4}$ nanoparticles (Figure 10); Raman spectra of the used $\mathrm{t}-\mathrm{ZnO}$ template and the fabricated aeronetworks (Figure S11); reversible buckling at central joint (Figure S12); reversible buckling of hollow tube (Figure S13); nanosails (Figure S14); list of wet-chemically prepared graphene-polymer composites and their electric properties (Table S1); and template-mediated assembly of $2 \mathrm{D}$ nanomaterials (PDF)

Wet-chemical infiltration of a highly porous (94\%) t$\mathrm{ZnO}$ template with an aqueous dispersion $\left(2 \mathrm{mg} \mathrm{mL}^{-1}\right)$ of GO (Video S1) (MP4) 
Guided movement of an aeromagnet with a permanent magnet (Video S2) (MP4)

Repeated compression of Aero-rGO at compression strain of 33\% (Video S3) (MP4)

Reversible bending of a hollow rGO microtube at central joint induced by a micromanipulator (Video S4) (MP4) Reversible buckling of a hollow rGO microtube induced by a micromanipulator (Video S5) (MP4)

\section{AUTHOR INFORMATION}

\section{Corresponding Author}

*E-mail: fas@tf.uni-kiel.de.

\section{ORCID}

Florian Rasch: 0000-0003-4623-1659

Fabian Schütt: 0000-0003-2942-503X

Oleksandr Polonskyi: 0000-0001-5013-0944

Martin R. Lohe: 0000-0001-5458-779X

Yogendra K. Mishra: 0000-0002-8786-9379

Franz Faupel: 0000-0003-3367-1655

Xinliang Feng: 0000-0003-3885-2703

\section{Author Contributions}

R.A., F.R., and F.S. came up with the concept. F.R., F.S., Y.K.M., M.R.L., A.S.N., R.A., F.F., L.K., and X.F. designed the study. F.R., F.S., and L.M.S. fabricated the samples. F.R., R.A., S.K., M.R.L., A.S.N., F.S., and L.M.S. analyzed the data. J.S. and L.K. carried out the TEM measurements and corresponding analysis. F.R. carried out SEM and Raman measurements. O.P. and F.F. carried out the XPS measurements and analyzed the data. F.R., F.S., and R.A. finalized the study and wrote the paper. All of the authors have contributed to the discussion of the results and reviewed the manuscript.

\section{Notes}

The authors declare no competing financial interest.

\section{ACKNOWLEDGMENTS}

The authors acknowledge funding by the Deutsche Forschungsgemeinschaft (DFG) under contracts $A D 183 / 27-1$, $\mathrm{AD} 183 / 18-1$ and GRK 2154. This project has received funding from the European Union's Horizon 2020 research and innovation programme under grant agreement No GrapheneCore2 785219.

\section{REFERENCES}

(1) Novoselov, K. S.; Geim, A. K.; Morozov, S. V.; Jiang, D.; Zhang, Y.; Dubonos, S. V.; Grigorieva, I. V.; Firsov, A. A. Electric Field Effect in Atomically Thin Carbon Films. Science 2004, 306, 666-669.

(2) Zhang, Y.; Tan, Y.-W.; Stormer, H. L.; Kim, P. Experimental Observation of the Quantum Hall Effect and Berry's Phase in Graphene. Nature 2005, 438, 201-204.

(3) Novoselov, K. S.; Geim, A. K.; Morozov, S. V.; Jiang, D.; Katsnelson, M. I.; Grigorieva, I. V.; Dubonos, S. V.; Firsov, A. A. TwoDimensional Gas of Massless Dirac Fermions in Graphene. Nature 2005, 438, 197-200.

(4) Lee, C.; Wei, X.; Kysar, J. W.; Hone, J. Measurement of the Elastic Properties and Intrinsic Strength of Monolayer Graphene. Science 2008, 321, 385-388.

(5) Balandin, A. A.; Ghosh, S.; Bao, W.; Calizo, I.; Teweldebrhan, D.; Miao, F.; Lau, C. N. Superior Thermal Conductivity of SingleLayer Graphene. Nano Lett. 2008, 8, 902-907.

(6) Lupan, O.; Postica, V.; Mecklenburg, M.; Schulte, K.; Mishra, Y. K.; Fiedler, B.; Adelung, R. Low Powered, Tunable and Ultra-Light Aerographite Sensor for Climate Relevant Gas Monitoring. J. Mater. Chem. A 2016, 4, 16723-16730.
(7) Lupan, O.; Schütt, F.; Postica, V.; Smazna, D.; Mishra, Y. K.; Adelung, R. Sensing Performances of Pure and Hybridized Carbon Nanotubes-ZnO Nanowire Networks: A Detailed Study. Sci. Rep. 2017, 7, No. 14715.

(8) Reddy, A. L. M.; Srivastava, A.; Gowda, S. R.; Gullapalli, H.; Dubey, M.; Ajayan, P. M. Synthesis Of Nitrogen-Doped Graphene Films For Lithium Battery Application. ACS Nano 2010, 4, 63376342.

(9) El-Kady, M. F.; Shao, Y.; Kaner, R. B. Graphene for Batteries, Supercapacitors and Beyond. Nat. Rev. Mater. 2016, 1, No. 16033.

(10) Parlak, O.; Kumar Mishra, Y.; Grigoriev, A.; Mecklenburg, M.; Luo, W.; Keene, S.; Salleo, A.; Schulte, K.; Ahuja, R.; Adelung, R.; et al. Hierarchical Aerographite Nano-Microtubular Tetrapodal Networks Based Electrodes as Lightweight Supercapacitor. Nano Energy 2017, 34, 570-577.

(11) Cao, X.; Shi, Y.; Shi, W.; Lu, G.; Huang, X.; Yan, Q.; Zhang, Q.; Zhang, H. Preparation of Novel 3D Graphene Networks for Supercapacitor Applications. Small 2011, 7, 3163-3168.

(12) Shehzad, K.; Xu, Y.; Gao, C.; Duan, X. Three-Dimensional Macro-Structures of Two-Dimensional Nanomaterials. Chem. Soc. Rev. 2016, 45, 5541-5588.

(13) Chen, Z.; Ren, W.; Gao, L.; Liu, B.; Pei, S.; Cheng, H.-M. Three-Dimensional Flexible and Conductive Interconnected Graphene Networks Grown by Chemical Vapour Deposition. Nat. Mater. 2011, 10, 424-428.

(14) Ning, J.; Xu, X.; Liu, C.; Fan, D. L. Three-Dimensional Multilevel Porous Thin Graphite Nanosuperstructures for $\mathrm{Ni}(\mathrm{OH})_{2}$ -Based Energy Storage Devices. J. Mater. Chem. A 2014, 2, 1576815773.

(15) Qiu, L.; Liu, J. Z.; Chang, S. L. Y.; Wu, Y.; Li, D. Biomimetic Superelastic Graphene-Based Cellular Monoliths. Nat. Commun. 2012, 3, No. 1241.

(16) Wang, X.; Zhang, Y.; Zhi, C.; Wang, X.; Tang, D.; Xu, Y.; Weng, Q.; Jiang, X.; Mitome, M.; Golberg, D.; et al. ThreeDimensional Strutted Graphene Grown by Substrate-Free Sugar Blowing for High-Power-Density Supercapacitors. Nat. Commun. 2013, 4, No. 2905.

(17) Srinivas, G.; Burress, J. W.; Ford, J.; Yildirim, T. Porous Graphene Oxide Frameworks: Synthesis and Gas Sorption Properties. J. Mater. Chem. 2011, 21, 11323.

(18) Xu, Y.; Sheng, K.; Li, C.; Shi, G. Self-Assembled Graphene Hydrogel via a One-Step Hydrothermal Process. ACS Nano 2010, 4, 4324-4330.

(19) Vickery, J. L.; Patil, A. J.; Mann, S. Fabrication of GraphenePolymer Nanocomposites With Higher-Order Three-Dimensional Architectures. Adv. Mater. 2009, 21, 2180-2184.

(20) Bai, H.; Li, C.; Wang, X.; Shi, G. A PH-Sensitive Graphene Oxide Composite Hydrogel. Chem. Commun. 2010, 46, 2376.

(21) Sun, H.; Xu, Z.; Gao, C. Multifunctional, Ultra-Flyweight, Synergistically Assembled Carbon Aerogels. Adv. Mater. 2013, 25, 2554-2560.

(22) Chen, M.; Zhang, C.; Li, X.; Zhang, L. L.; Ma, Y.; Zhang, L. L.; Xu, X.; Xia, F.; Wang, W.; Gao, J. A One-Step Method for Reduction and Self-Assembling of Graphene Oxide into Reduced Graphene Oxide Aerogels. J. Mater. Chem. A 2013, 1, 2869.

(23) Taale, M.; Schütt, F.; Carey, T.; Marx, J.; Mishra, Y. K.; Stock, N.; Fiedler, B.; Torrisi, F.; Adelung, R.; Selhuber-Unkel, C. Biomimetic Carbon Fiber Systems Engineering: A Modular Design Strategy To Generate Biofunctional Composites from Graphene and Carbon Nanofibers. ACS Appl. Mater. Interfaces 2019, 11, 5325-5335.

(24) Taale, M.; Schütt, F.; Zheng, K.; Mishra, Y. K.; Boccaccini, A. R.; Adelung, R.; Selhuber-Unkel, C. Bioactive Carbon-Based Hybrid 3D Scaffolds for Osteoblast Growth. ACS Appl. Mater. Interfaces 2018, 10, 43874-43886.

(25) Schütt, F.; Postica, V.; Adelung, R.; Lupan, O. Single and Networked ZnO-CNT Hybrid Tetrapods for Selective RoomTemperature High-Performance Ammonia Sensors. ACS Appl. Mater. Interfaces 2017, 9, 23107-23118. 
(26) Schütt, F.; Signetti, S.; Krüger, H.; Röder, S.; Smazna, D.; Kaps, S.; Gorb, S. N.; Mishra, Y. K.; Pugno, N. M.; Adelung, R. Hierarchical Self-Entangled Carbon Nanotube Tube Networks. Nat. Commun. 2017, 8, No. 1215.

(27) Xiao, J.; Mei, D.; Li, X.; Xu, W.; Wang, D.; Graff, G. L.; Bennett, W. D.; Nie, Z.; Saraf, L. V.; Aksay, I. A.; et al. Hierarchically Porous Graphene as a Lithium-Air Battery Electrode. Nano Lett. 2011, 11, 5071-5078.

(28) Li, N.; Zhang, Q.; Gao, S.; Song, Q.; Huang, R.; Wang, L.; Liu, L.; Dai, J.; Tang, M.; Cheng, G. Three-Dimensional Graphene Foam as a Biocompatible and Conductive Scaffold for Neural Stem Cells. Sci. Rep. 2013, 3, No. 1604.

(29) Mishra, Y. K.; Modi, G.; Cretu, V.; Postica, V.; Lupan, O.; Reimer, T.; Paulowicz, I.; Hrkac, V.; Benecke, W.; Kienle, L.; et al. Direct Growth of Freestanding ZnO Tetrapod Networks for Multifunctional Applications in Photocatalysis, UV Photodetection, and Gas Sensing. ACS Appl. Mater. Interfaces 2015, 7, 14303-14316.

(30) Mishra, Y. K.; Kaps, S.; Schuchardt, A.; Paulowicz, I.; Jin, X.; Gedamu, D.; Wille, S.; Lupan, O.; Adelung, R. Versatile Fabrication of Complex Shaped Metal Oxide Nano-Microstructures and Their Interconnected Networks for Multifunctional Applications. KONA Powder Part. J. 2014, 31, 92-110.

(31) Mishra, Y. K.; Kaps, S.; Schuchardt, A.; Paulowicz, I.; Jin, X.; Gedamu, D.; Freitag, S.; Claus, M.; Wille, S.; Kovalev, A.; et al. Fabrication of Macroscopically Flexible and Highly Porous 3D Semiconductor Networks from Interpenetrating Nanostructures by a Simple Flame Transport Approach. Part. Part. Syst. Charact. 2013, 30, $775-783$.

(32) Worsley, M. A.; Shin, S. J.; Merrill, M. D.; Lenhardt, J.; Nelson, A. J.; Woo, L. Y.; Gash, A. E.; Baumann, T. F.; Orme, C. A. Ultralow Density, Monolithic $\mathrm{WS}_{2}, \mathrm{MoS}_{2}$, and $\mathrm{MoS}_{2} /$ Graphene Aerogels. ACS Nano 2015, 9, 4698-4705.

(33) Sui, Z.-Y.; Cui, Y.; Zhu, J.-H.; Han, B.-H. Preparation of ThreeDimensional Graphene Oxide-Polyethylenimine Porous Materials as Dye and Gas Adsorbents. ACS Appl. Mater. Interfaces 2013, 5, 91729179.

(34) Fan, D.; Liu, Y.; He, J.; Zhou, Y.; Yang, Y. Porous GrapheneBased Materials by Thermolytic Cracking. J. Mater. Chem. 2012, 22, 1396-1402.

(35) Huang, X.; Qian, K.; Yang, J.; Zhang, J.; Li, L.; Yu, C.; Zhao, D. Functional Nanoporous Graphene Foams with Controlled Pore Sizes. Adv. Mater. 2012, 24, 4419-4423.

(36) Mishra, Y. K.; Adelung, R. ZnO Tetrapod Materials for Functional Applications. Mater. Today 2018, 21, 631-651.

(37) Wu, S.; Xiong, G.; Yang, H.; Tian, Y.; Gong, B.; Wan, H.; Wang, Y.; Fisher, T. S.; Yan, J.; Cen, K.; et al. Scalable Production of Integrated Graphene Nanoarchitectures for Ultrafast Solar-Thermal Conversion and Vapor Generation. Matter 2019, 1, 1017-1032.

(38) Pei, S.; Cheng, H.-M. The Reduction of Graphene Oxide. Carbon 2012, 50, 3210-3228.

(39) Parvez, K.; Wu, Z.-S.; Li, R.; Liu, X.; Graf, R.; Feng, X.; Müllen, K. Exfoliation of Graphite into Graphene in Aqueous Solutions of Inorganic Salts. J. Am. Chem. Soc. 2014, 136, 6083-6091.

(40) Ferrari, A. C.; Meyer, J. C.; Scardaci, V.; Casiraghi, C.; Lazzeri, M.; Mauri, F.; Piscanec, S.; Jiang, D.; Novoselov, K. S.; Roth, S.; et al. Raman Spectrum of Graphene and Graphene Layers. Phys. Rev. Lett. 2006, 97, No. 187401.

(41) Tuinstra, F.; Koenig, J. L. Raman Spectrum of Graphite. J. Chem. Phys. 1970, 53, 1126-1130.

(42) Moon, I. K.; Lee, J.; Ruoff, R. S.; Lee, H. Reduced Graphene Oxide by Chemical Graphitization. Nat. Commun. 2010, 1, No. 73.

(43) Yang, D.; Velamakanni, A.; Bozoklu, G.; Park, S.; Stoller, M.; Piner, R. D.; Stankovich, S.; Jung, I.; Field, D. A.; Ventrice, C. A.; et al. Chemical Analysis of Graphene Oxide Films after Heat and Chemical Treatments by X-Ray Photoelectron and Micro-Raman Spectroscopy. Carbon 2009, 47, 145-152.

(44) Chen, W.; Yan, L.; Bangal, P. R. Preparation of Graphene by the Rapid and Mild Thermal Reduction of Graphene Oxide Induced by Microwaves. Carbon 2010, 48, 1146-1152.
(45) Stankovich, S.; Dikin, D. A.; Piner, R. D.; Kohlhaas, K. A.; Kleinhammes, A.; Jia, Y.; Wu, Y.; Nguyen, S. T.; Ruoff, R. S. Synthesis of Graphene-Based Nanosheets via Chemical Reduction of Exfoliated Graphite Oxide. Carbon 2007, 45, 1558-1565.

(46) Ferrari, A. C.; Robertson, J. Interpretation of Raman Spectra of Disordered and Amorphous Carbon. Phys. Rev. B 2000, 61, 1409514107.

(47) Paredes, J. I.; Villar-Rodil, S.; Solís-Fernández, P.; MartínezAlonso, A.; Tascón, J. M. D. Atomic Force and Scanning Tunneling Microscopy Imaging of Graphene Nanosheets Derived from Graphite Oxide. Langmuir 2009, 25, 5957-5968.

(48) Chieu, T. C.; Dresselhaus, M. S.; Endo, M. Raman Studies of Benzene-Derived Graphite Fibers. Phys. Rev. B 1982, 26, 5867-5877.

(49) Ewels, P.; Sikora, T.; Serin, V.; Ewels, C. P.; Lajaunie, L. A Complete Overhaul of the Electron Energy-Loss Spectroscopy and XRay Absorption Spectroscopy Database: Eelsdb.Eu. Microsc. Microanal. 2016, 22, 717-724.

(50) Gass, M. H.; Bangert, U.; Bleloch, A. L.; Wang, P.; Nair, R. R.; Geim, A. K. Free-Standing Graphene at Atomic Resolution. Nat. Nanotechnol. 2008, 3, 676-681.

(51) Malis, T.; Cheng, S. C.; Egerton, R. F. EELS Log-Ratio Technique for Specimen-Thickness Measurement in the TEM. J. Electron Microsc. Tech. 1988, 8, 193-200.

(52) Wu, T.; Chen, M.; Zhang, L.; Xu, X.; Liu, Y.; Yan, J.; Wang, W.; Gao, J. Three-Dimensional Graphene-Based Aerogels Prepared by a Self-Assembly Process and Its Excellent Catalytic and Absorbing Performance. J. Mater. Chem. A 2013, 1, 7612.

(53) Kim, F.; Cote, L. J.; Huang, J. Graphene Oxide: Surface Activity and Two-Dimensional Assembly. Adv. Mater. 2010, 22, 1954-1958.

(54) Marsden, A. J.; Papageorgiou, D. G.; Vallés, C.; Liscio, A.; Palermo, V.; Bissett, M. A.; Young, R. J.; Kinloch, I. A. Electrical Percolation in Graphene-Polymer Composites. 2D Mater. 2018, 5, No. 032003.

(55) Bauhofer, W.; Kovacs, J. Z. A Review and Analysis of Electrical Percolation in Carbon Nanotube Polymer Composites. Compos. Sci. Technol. 2009, 69, 1486-1498.

(56) Yousefi, N.; Sun, X.; Lin, X.; Shen, X.; Jia, J.; Zhang, B.; Tang, B.; Chan, M.; Kim, J.-K. Highly Aligned Graphene/Polymer Nanocomposites with Excellent Dielectric Properties for HighPerformance Electromagnetic Interference Shielding. Adv. Mater. 2014, 26, 5480-5487.

(57) Lan, Y.; Liu, H.; Cao, X.; Zhao, S.; Dai, K.; Yan, X.; Zheng, G.; Liu, C.; Shen, C.; Guo, Z. Electrically Conductive Thermoplastic Polyurethane/Polypropylene Nanocomposites with Selectively Distributed Graphene. Polymer 2016, 97, 11-19.

(58) Li, N.; Chen, Z.; Ren, W.; Li, F.; Cheng, H.-M. Flexible Graphene-Based Lithium Ion Batteries with Ultrafast Charge and Discharge Rates. Proc. Natl. Acad. Sci. U.S.A. 2012, 17360-17365.

(59) Bi, H.; Chen, I.-W.; Lin, T.; Huang, F. A New Tubular Graphene Form of a Tetrahedrally Connected Cellular Structure. Adv. Mater. 2015, 27, 5943-5949.

(60) Suhr, J.; Victor, P.; Ci, L.; Sreekala, S.; Zhang, X.; Nalamasu, O.; Ajayan, P. M. Fatigue Resistance of Aligned Carbon Nanotube Arrays under Cyclic Compression. Nat. Nanotechnol. 2007, 2, 417421.

(61) Zhuo, H.; Hu, Y.; Tong, X.; Chen, Z.; Zhong, L.; Lai, H.; Liu, L.; Jing, S.; Liu, Q.; Liu, C.; et al. A Supercompressible, Elastic, and Bendable Carbon Aerogel with Ultrasensitive Detection Limits for Compression Strain, Pressure, and Bending Angle. Adv. Mater. 2018, 30, No. 1706705.

(62) Falvo, M. R.; Clary, G. J.; Taylor, R. M.; Chi, V.; Brooks, F. P.; Washburn, S.; Superfine, R. Bending and Buckling of Carbon Nanotubes under Large Strain. Nature 1997, 389, 582-584.

(63) Meija, R.; Signetti, S.; Schuchardt, A.; Meurisch, K.; Smazna, D.; Mecklenburg, M.; Schulte, K.; Erts, D.; Lupan, O.; Fiedler, B.; et al. Nanomechanics of Individual Aerographite Tetrapods. Nat. Commun. 2017, 8, No. 14982. 
(64) Xu, X.; Zhang, Q.; Yu, Y.; Chen, W.; Hu, H.; Li, H. Naturally Dried Graphene Aerogels with Superelasticity and Tunable Poisson's Ratio. Adv. Mater. 2016, 28, 9223-9230.

(65) Wang, C.; Pan, D.; Chen, S. Energy Dissipative Mechanism of Graphene Foam Materials. Carbon 2018, 132, 641-650.

(66) Wang, X.; Lu, L.-L.; Yu, Z.-L.; Xu, X.-W.; Zheng, Y.-R.; Yu, S.H. Scalable Template Synthesis of Resorcinol-Formaldehyde/ Graphene Oxide Composite Aerogels with Tunable Densities and Mechanical Properties. Angew. Chem., Int. Ed. 2015, 54, 2397-2401. (67) Shaygan Nia, A.; Rana, S.; Döhler, D.; Jirsa, F.; Meister, A.; Guadagno, L.; Koslowski, E.; Bron, M.; Binder, W. H. CarbonSupported Copper Nanomaterials: Recyclable Catalysts for Huisgen $[3+2]$ Cycloaddition Reactions. Chem. - Eur. J. 2015, 21, 1076310770.

(68) Shaygan Nia, A.; Rana, S.; Döhler, D.; Noirfalise, X.; Belfiore, A.; Binder, W. H. Click Chemistry Promoted by Graphene Supported Copper Nanomaterials. Chem. Commun. 2014, 50, 15374-15377. 